\title{
Predictive value of serial measurements of quality of life on all-cause mortality in prostate cancer patients: data from CaPSURE ${ }^{\mathrm{TM}}$ (cancer of the prostate strategic urologic research endeavor) database
}

\author{
Natalia Sadetsky · Alan Hubbard · Peter R. Carroll • \\ William Satariano
}

Accepted: 3 August 2009/Published online: 21 August 2009

(C) The Author(s) 2009. This article is published with open access at Springerlink.com

\begin{abstract}
Introduction Health-related quality of life (HRQOL) is a legitimate construct for evaluating treatment and its side effects. Recently, predictive value of HRQOL on survival also has been of interest. In light of the longer survival in patients with prostate cancer and importance of quality of life, we seek to evaluate the association between HRQOL and survival using traditional and novel techniques.

Methods Patients from CaPSURE (Cancer of the Prostate Strategic Urologic Research Endeavor) who were treated within 6 months of diagnosis and had pre-treatment and sufficient post-treatment follow-up information constituted the study population. A sample consisting of 2,899 patients met the study criteria. SF-36 domains were used to measure HRQOL outcomes. Categorical variables were created for HRQOL based on the baseline distribution of the lower 10th percentile and the remainder of the patients. Association between HRQOL and survival (defined by all-cause mortality) in patients with prostate cancer was evaluated using Cox proportional hazards models controlling for age at diagnosis, type of treatment received, clinical risk classification, and number of comorbidities. Sequential bootstrap resampling was implemented to evaluate stability of the model. Univariate and multivariate Cox proportional hazards models were fit using various time points over the course of follow-up.

Results In the analysis looking at association of HRQOL baseline measurements, higher levels of physical function and general health were significantly associated with better survival (HR 0.49 95\% CI $0.32-0.78$ and HR 0.51 95\% CI $0.35-0.75$, respectively). Post-treatment analysis
\end{abstract}

N. Sadetsky $(\bowtie) \cdot$ A. Hubbard · P. R. Carroll · W. Satariano UCSF, San Francisco, CA, USA

e-mail: nsadetsky@urology.ucsf.edu demonstrated similar results. In time-dependent analysis, higher levels of physical function, role physical, and general health were significantly associated with better survival (HR ranged from 0.57 to 0.65 ). In addition, analysis looking at change in HRQOL scores demonstrated an association between higher scores on physical function, role physical, vitality, social function, and general health and longer survival (HR ranged from 0.56 to 0.63 ).

Conclusion This study demonstrated that several domains of HRQOL were significantly associated with survival in a large group of patients with localized prostate cancer. This association was maintained over the course of disease regardless of the time of the assessment. Results from our study have both research and clinical relevance. They could provide information that enable us to not only improve communication with patients and families, but also to develop interventions and treatments best suited for the patient.

Keywords Prostate cancer - Quality of life - Survival

\section{Introduction}

The burden that disease places on patients, families, and society has become an important consideration in health care [1]. With more people living with chronic conditions, cancer included, quality of life, in addition to the traditional outcomes such as morbidity and mortality, becomes an important factor in decision-making interventions and program development [2].

With improvements in diagnosis and treatment, there has been a marked increase in long-term cancer survivors in the past decade [3]. Although numerous studies exist on both general and disease-related quality of life in cancer 
survivors, most of the research covers periods close to diagnosis and treatment. A variety of factors such as a patient's clinical and sociodemographic characteristics, level of function prior to treatment, and type and side effects of treatment could influence survival over the course of disease. Moreover, predictive value of quality of life measured at various points over the course of disease could provide information on complex pathways between physical and psychological manifestations of disease. Recently, numerous studies addressed the question of predictive value of HRQOL in survival [4-12]. It has been shown that HRQOL can independently predict survival in several types of diseases, independent of clinical and demographic characteristics of the patient. In a majority of studies, HRQOL measures assessed at baseline (i.e. before treatment started) demonstrated a strong association with survival for a variety of conditions, ranging from advanced lung carcinoma, breast carcinoma, multiple myeloma, melanoma, esophageal carcinoma, head and neck carcinoma, and advanced prostate cancer, while some of the studies report no association [4-11, 13].

In the last decade, there has been an upsurge of HRQOL research in the field of prostate cancer. Controversy surrounding screening, diagnosis, and treatment of prostate cancer indicates the particular importance of quality of life measurements. Evaluations of HRQOL outcomes in prostate cancer treatments have focused on the specific areas of functioning rather than broader physical, emotional, and social well-being. Studies evaluating specific outcomes after treatment concluded that sexual function declines rapidly after radical prostatectomy with eventual improvement, while external beam radiation produce smaller but more prolonged impairments $[14,15]$. However, there have been a limited number of the studies evaluating predictive value of HRQOL and survival in prostate cancer patients, with the majority of them examining patients with metastatic prostate cancer. In the study by Collette et al., baseline HRQOL scores were associated with survival in 391 patients with metastatic disease. However, the investigators concluded that the addition of HRQOL variables did not improve the predictive power of clinical and biochemical factors on survival models [5]. Sullivan and colleagues described the relationship between HRQOL measurements at baseline, 4, and 12 weeks in 809 patients and determined that both baseline and change scores were significantly associated with a variety of outcomes [11].

As life expectancy increases in patients with clinically localized prostate cancer, evaluation of the long-term effects of treatment on survival becomes more imperative. At the same time, the absence of sufficient clinical and treatment-related quality of life follow-up in large populations of patients with localized disease is a major obstacle in assessing changes of QOL over time. Numerous clinical and psychological factors, such as diagnosis, treatment, recovery, and side effects could influence a patient's own health perception and affect his general health over time. Longitudinal studies of prostate cancer have shown that a patient's QOL scores are likely to change over the course of follow-up, and failure to examine trends across numerous consecutive time points could lead to underestimation of true associations between HRQOL and clinical outcomes [16-18]. Several studies have addressed the dynamic relationship between serial measurements of HRQOL and survival, demonstrating that results over time differ from cross-sectional assessments. In the study by Ferraro and Kelley-Moore, the association between self-reported health (SHR) and mortality was demonstrated only when SHR measurements were treated as time-dependent covariates [17]. At the same time, in the analysis by Lyyra et al., use of time-dependent Cox proportional hazards models enabled investigators to demonstrate increased association of SHR and mortality in both men and women [16]. Given longer survival of patients with prostate cancer and greater emphasis on improving quality over quantity of life, it is important to incorporate the time-dependent nature of HRQOL into prostate cancer research.

Although studies reporting predictive effects of HRQOL on survival have been plentiful, there are several factors that could make interpretation of results difficult. Insufficient adjustment for known clinical prognostic factors and ignoring correlations between scales used to measure HRQOL are just two of them. A recent paper by Van Steen et al. discussed these issues [19]. In addition, methods to address those issues in the context of Cox Regression models have been developed by Sauerbrei and Schumacher [20].

In the current study, traditional analysis of the association between HRQOL and survival is enhanced by bootstrap resampling techniques that are used in effort to avoid possible biases in selection of variables for each model, and implementation of the time-dependent survival analysis to account for the dynamic nature of HRQOL.

The goal of the present study was to assess whether HRQOL (as both a constant and a time-dependent covariate) measured (1) at baseline, (2) at post-treatment, and (3) as change between baseline and the time preceding outcomes (death) is associated with survival.

\section{Materials and methods}

Study population

CaPSURE $^{\mathrm{TM}}$ (Cancer of the Prostate Strategic Urologic Research Endeavor) patients are recruited from 40 community-based, academic, and veterans affairs (VA) urology 
practices across the United States by participating urologists who report clinical data and follow-up information on diagnostic tests and treatments. Approximately $80 \%$ of patients are drawn from community-based practices in 25 states, ensuring a broad representation of geographically diverse community patients. HRQOL data are obtained from a self-administered questionnaire sent to each patient's home biannually. Patients are treated according to their physicians' usual practices and are followed until time of death or withdrawal from the study. Detailed descriptions of the CaPSURE study populations and methods have been published previously [21, 22].

Men who were newly diagnosed at entry to CaPSURE had information on initial treatment and had serial (baseline, immediate post-treatment, and at least one longer post-treatment) quality of life assessments made up the study population. As of June 2007, 13,124 patients were enrolled in CaPSURE. Of these, 8,667 were newly diagnosed (i.e. enrolled within 6 months of diagnosis) and had information on initial treatment; and 2,899 had HRQOL assessments prior to treatment as well as within 2 years after treatment.

\section{Outcomes measured}

General health-related quality of life (HRQOL) was measured using RAND 36-item (SF-36), version 1.0 health survey. This instrument includes eight individual domains, four physical and four mental: physical functioning (PF), role limitation because of physical problems (RP), bodily pain $(\mathrm{BP})$, general health perception $(\mathrm{GH})$, role limitations because of emotional problems (RE), energy/fatigue (VT), emotional well-being (MH), and social functioning (SO) and two summary scores, measuring physical and mental components [23]. Results of each score are standardized to go from 0 to 100 , with higher scores representing better outcomes. For ease of interpretation, categorical variables were created for HRQOL based on the baseline distribution of the lower 10th percentile and the remainder of the patients. In addition, continuous values of HRQOL outcomes were tested.

All-cause and disease-specific mortality were reported by treating physicians or next of kin and confirmed by death certificates or national death index (NDI) when the death certificates were unavailable.

Type of treatment was defined as radical prostatectomy (RP)-surgical removal of prostate, external beam radiation (XRT), cryotherapy (Cryo), and hormonal treatment (HT). Watchful waiting patients were not included in study population due to small sample size and insufficient follow-up data.

Demographic and clinical characteristics of the study population such as race/ethnicity, BMI, and marital status were evaluated in initial analysis. They were not included in the main analysis due to poor distribution of values in the study population or irrelevance to the research question.

Age at diagnosis was included as both continuous and a categorical variable with four levels: $<55$ years old, 5564 years old, 65-74 years old, and $>75$ years old. Number of comorbidities at baseline was defined as a categorical variable with three levels: no comorbidities, one to three, and greater than three. Pre-treatment clinical stage, Gleason grade, and prostate-specific antigen (PSA) level were combined into prostate cancer risk categories, as described by D'Amico et al. [24]: low risk (stage T1 or 2a, Gleason score 2-6, PSA level $<10 \mathrm{ng} / \mathrm{mL}$ ), intermediate risk (stage T2b or Gleason score 7 or PSA between 10 and $20 \mathrm{ng} / \mathrm{mL}$ ), and high risk (any stage greater than T2b, Gleason score 8-10, PSA level $>20 \mathrm{ng} / \mathrm{mL}$ ). In addition, separate components of risk classification categories.

Statistical methods

Association between HRQOL and survival in patients with prostate cancer was evaluated using Cox proportional hazards models to estimate hazard ratios (HR) that presents a relative risk of survival between groups with different values of baseline HRQOL.

Sequential bootstrap resampling was implemented to identify the most stable variables associated with survival. This process was modeled on the research by MessingerRapport et al. [25] and methods developed by Sauerbrei and Schumacher [20]. This method incorporates stepwise selection with data-dependent methods of choosing variables. It gives an ability to evaluate both the distribution and contribution of each variable to the stability of a model, and could help in evaluation of the different stages of significance. Bootstrap resampling involved creating new random datasets from the original dataset with each dataset then undergoing Cox analysis with stepwise selection. The process was repeated 1,000 times, and the percentage of each variable included in the analysis was calculated. Those variables that entered the models at least $50 \%$ of the times are used for the final Cox analysis to estimate $\mathrm{HR}$ and $95 \%$ confidence intervals (CI).

Initially, univariate Cox proportional hazards model were fit for each baseline HRQOL outcome to determine which were related to overall survival.

Then, a series of multivariate models that included all HRQOL domains, type of initial treatment, prostate cancer disease severity, age at diagnosis, and number of comorbidities (chosen a priori for initial analysis) were used to determine the effect of HRQOL on survival at various time points over the course of disease. It should be noted that inferences from estimates in our models could be biased since they are based on the assumptions that the model is 
defined by choice of 'correct' variables; thus, this inference should be considered informal.

First, the associations of the baseline measurements (i.e. measurements reported prior to treatment) and overall survival were evaluated. Second, measurements reported in the first year after treatments were evaluated in a similar model. Third, to account for changes in HRQOL over time, a Cox proportional hazards regression model with timedependent covariates was run [26]. This method allowed the calculation of hazards at specific times that depended on the values of other covariates in the model [27]. Fourth, to account for changes in HRQOL, differences between initial and last HRQOL assessments, defined as either positive (improvement in HRQOL score) or negative (decline in HRQOL score) change, were evaluated.

Difference in $-2 \mathrm{LL}$ statistics were considered to assess the predictive value of the models with and without HRQOL for survival.

All analyses were performed using version 9.2 of SAS for Windows (SAS Institute, Cary, NC).

\section{Results}

The study population consisted of 2,899 patients. More than half of the patients underwent radical prostatectomy as an initial treatment (58\%), with brachytherapy and external beam radiation constituting another third (22 and $11 \%$, respectively), and the rest of treatments divided between cryosurgery and hormonal treatment. Most of the patients were between 55 and 74 years of age (77\%) with the rest equally divided between a younger and an older group $(<55$ and $>75)$. Mean follow-up time from treatment to either death or last contact was 41.7 months (Q1-24.12, Q3-56.6). Of the study population, 173 (6\%) have died with $29(1 \%)$ of them dying from prostate cancer. A higher proportion of deceased was treated with hormonal treatment and external beam radiation (23.75 and $12.35 \%$, respectively) with the remainder divided between brachytherapy and radical prostatectomy. Clinical and sociodemographic characteristics of study population are reported in Table 1. Mean values of baseline and post-treatment HRQOL are listed in Table 2.

Sequential multivariate Cox regression analysis was performed using bootstrap resampling to identify the most stable model. Variables that were entered into the models more than $50 \%$ at the time $(500$ out of 1,000$)$ were used for the final models. Subsequent analysis with variables selected through bootstrapping procedures revealed that several HRQOL measures are associated with survival even after adjusting for clinical severity, age, type of treatment received, and number of comorbidities.
Table 1 Clinical and sociodemographic characteristics of study population

\begin{tabular}{|c|c|c|c|}
\hline Study characteristic & Value & $N$ & $\%$ \\
\hline \multirow[t]{4}{*}{ Age at diagnosis } & $<55$ & 314 & 11 \\
\hline & $55-64$ & 1,031 & 36 \\
\hline & $65-74$ & 1,192 & 41 \\
\hline & $75+$ & 362 & 12 \\
\hline \multirow[t]{5}{*}{ Initial treatment } & Prostatectomy (RP) & 1,682 & 58 \\
\hline & Cryotherapy & 128 & 4 \\
\hline & Brachytherapy & 605 & 21 \\
\hline & External beam radiation (XRT) & 324 & 11 \\
\hline & Hormonal therapy (HT) & 160 & 6 \\
\hline \multirow[t]{3}{*}{ Clinical risk } & Low & 1,253 & 45 \\
\hline & Intermediate & 936 & 34 \\
\hline & High & 565 & 21 \\
\hline \multirow[t]{2}{*}{ Deceased } & No & 2,726 & 94 \\
\hline & Yes & 173 & 6 \\
\hline \multirow[t]{4}{*}{ BMI categories } & Normal $(<25.0)$ & 738 & 26 \\
\hline & Overweight (25.0-29.9) & 1,481 & 52 \\
\hline & Obese $(30-35)$ & 472 & 17 \\
\hline & Very Obese $(>35)$ & 141 & 5 \\
\hline \multirow[t]{2}{*}{ Race/Ethnicity } & White & 2,812 & 97 \\
\hline & Other & 87 & 3 \\
\hline \multirow[t]{5}{*}{ Income } & $<\$ 30,000$ & 673 & 23 \\
\hline & $\$ 30,000-50,000$ & 637 & 22 \\
\hline & $\$ 50,000-75,000$ & 526 & 18 \\
\hline & $>\$ 75,000$ & 800 & 28 \\
\hline & Unknown & 263 & 9 \\
\hline \multirow[t]{3}{*}{ Relationship } & In relationship & 2,623 & 90 \\
\hline & Single & 226 & 8 \\
\hline & Unknown & 50 & 2 \\
\hline
\end{tabular}

Baseline analysis

In the analysis looking at association of HRQOL measurements at baseline and survival, higher levels of physical function and general health were significantly associated with better survival (HR 0.49 95\% CI 0.32-0.78 and HR $0.5195 \%$ CI 0.35-0.75, respectively) (Figs. 1, 2).

Post-treatment analysis

Analyses were also conducted to examine the association between HRQOL measures at 1-year post-treatment and subsequent survival. Again, physical function and role physical at 1 year were associated with better survival (HR $0.4595 \%$ CI $0.29-0.67$ and HR $0.5595 \%$ CI $0.38-0.79$, respectively) (Figs. 3, 4). 
Table 2 Mean value of SF-36 scales over time

\begin{tabular}{|c|c|c|c|c|c|c|}
\hline HRQOL components SF-36 & $\begin{array}{l}\text { Baseline } \\
\text { Mean (SD) }\end{array}$ & $\begin{array}{l}\text { First year } \\
\text { Mean (SD) }\end{array}$ & $\begin{array}{l}\text { Second year } \\
\text { Mean (SD) }\end{array}$ & $\begin{array}{l}\text { Third year } \\
\text { Mean (SD) }\end{array}$ & $\begin{array}{l}\text { Forth year } \\
\text { Mean (SD) }\end{array}$ & $\begin{array}{l}\text { Fifth year } \\
\text { Mean (SD) }\end{array}$ \\
\hline Physical function & $85.90(20.17)$ & $81.54(22.29)$ & $84.36(21.41)$ & $83.71(21.95)$ & 83.35 (22.09) & $82.88(22.67)$ \\
\hline Role physical & $79.65(35.07)$ & $63.61(42.64)$ & $76.40(37.16)$ & $76.84(37.10)$ & $76.31(37.15)$ & $75.83(37.73)$ \\
\hline Role emotional & $83.65(31.74)$ & $82.91(33.16)$ & $86.79(29.31)$ & $87.01(29.39)$ & $87.87(28.37)$ & $88.58(27.55)$ \\
\hline Vitality & $67.08(19.32)$ & $62.57(20.36)$ & $65.43(19.95)$ & $65.46(20.20)$ & $65.82(20.47)$ & $65.89(20.47)$ \\
\hline Mental health & $78.86(16.03)$ & $80.62(15.52)$ & $81.31(15.04)$ & $81.63(15.12)$ & $81.79(15.05)$ & $82.28(14.54)$ \\
\hline Social function & $88.15(19.32)$ & $82.50(23.43)$ & $88.27(19.47)$ & $88.36(19.93)$ & $88.48(20.14)$ & $87.78(20.87)$ \\
\hline Bodily pain & $84.28(19.86)$ & $79.69(21.86)$ & $82.91(20.52)$ & $81.89(20.82)$ & $81.24(21.04)$ & $80.64(21.08)$ \\
\hline General health & $72.43(19.12)$ & $72.41(19.39)$ & $72.41(20.10)$ & $71.97(20.03)$ & $71.43(20.64)$ & $71.49(20.51)$ \\
\hline Physical component summary & $50.79(9.20)$ & $47.52(9.95)$ & $49.59(9.55)$ & $49.19(9.83)$ & $48.85(9.96)$ & $48.65(10.18)$ \\
\hline Mental component summary & $52.18(9.18)$ & $52.67(8.93)$ & $53.49(8.24)$ & $53.67(8.43)$ & $53.97(8.54)$ & $54.25(8.01)$ \\
\hline
\end{tabular}

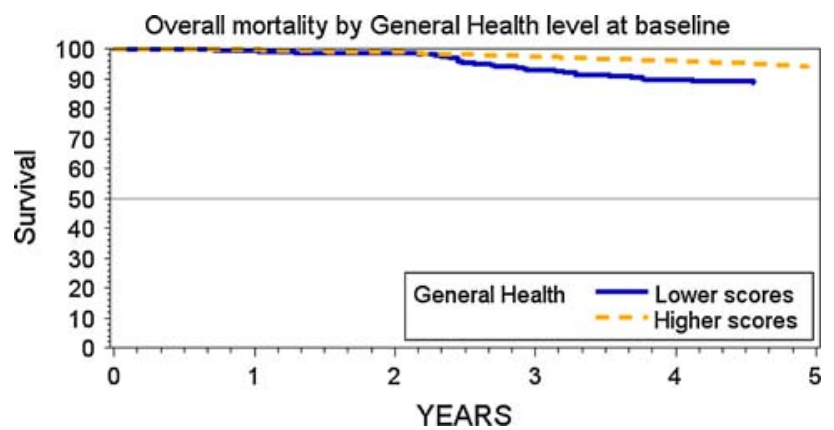

Fig. 1 Overall mortality by general health level at baseline

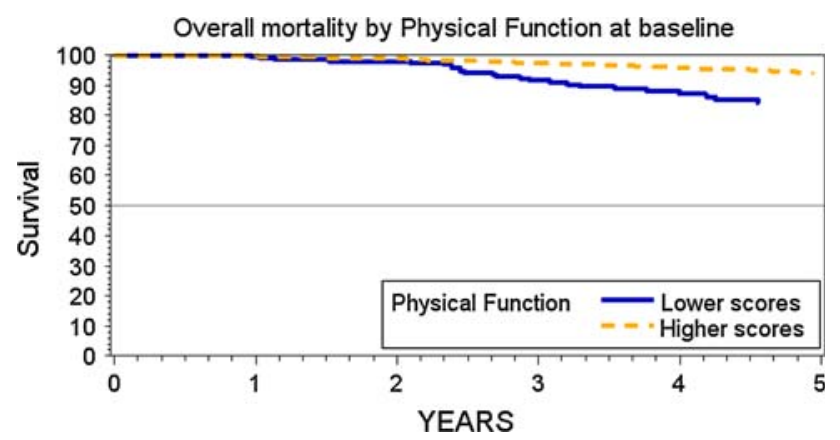

Fig. 2 Overall mortality by physical function at baseline

Time-dependent covariates

Further analysis was undertaken to account for HRQOL changes over time by using time-dependent Cox proportional hazards models. In this analysis, HRQOL was modeled as a variable that may change in value over time. It was assumed that changes in HRQOL prior to an event (either death or survival) would be highly associated with death. In this analysis, treating HRQOL as a time-dependent covariate, higher levels of physical function, role physical, and general health were significantly associated with better survival (HR ranged from 0.57 to 0.65 ).

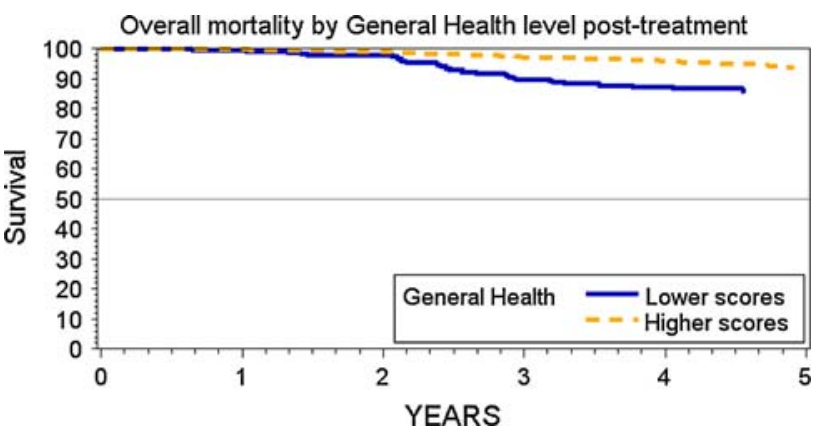

Fig. 3 Overall mortality by general health level post-treatment

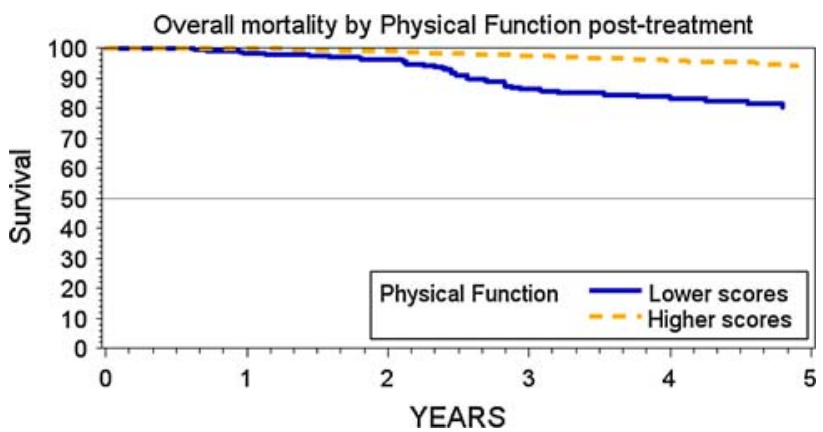

Fig. 4 Overall mortality by physical function post-treatment

\section{Change in HRQOL}

Analysis was undertaken using either improvement or decline in HRQOL scores between initial and last HRQOL assessments. In this analysis, improvement in social function and general health were associated with higher survival (HR 0.64 95\% CI 0.43-0.94 and HR 0.55 95\% CI $0.37-0.82$, respectively). A summary of the results of multivariate analysis are listed in Table 3.

In addition, age (evaluated as both categorical and continuous measure) and disease severity (evaluated as D'Amico categories and by inclusion of all components of 
Table 3 Results from multivariate Cox regression model (a) baseline, (b) time-dependent analysis, and (c) decline in HRQOL between baseline and last assessment

\begin{tabular}{ll}
\hline Parameter & Hazard ratio 95\% confidence limits
\end{tabular}

(a)

Physical function

Lower $10 \%$ percentile

Referent

General health

Remainder $90 \%$

$0.51(0.277-0.936)$

Lower $10 \%$ percentile

Referent

Age

Remainder $90 \%$

0.35 (0.194-0.628)

$<55$

Referent

$55-64$

$1.88(0.72-4.86)$

65-74

$2.84(1.12-7.22)$

$75+$

$2.91(1.08-7.02)$

Clinical risk

Low

Referent

Intermediate

$1.29(0.615-2.703)$

High

$2.78(1.408-5.471)$

Parameter

Hazard ratio $95 \%$ confidence limits

(b)

Physical function

Lower $10 \%$ percentile

Referent

Remainder $90 \%$

0.52 (0.34-0.77)

Role physical

Lower $10 \%$ percentile

Referent

Remainder $90 \%$

0.43 (0.29-0.65)

General health

Lower $10 \%$ percentile

Referent

Remainder $90 \%$

$0.35(0.24-0.52)$

Age

$<55$

Referent

55-64

2.43 (0.85-6.92)

65-74

$3.76(1.37-10.32)$

$75+$

4.90 (1.74-13.78)

Clinical risk

Low

Referent

Intermediate

1.35 (0.88-2.07)

High

$2.02(1.32-3.10)$

Variables

Value

Hazard ratio $95 \%$ confidence limits

(c)

Social function

Decline

Referent

General function

Improvement

0.64 (0.43-0.94)

Decline

Referent

Improvement

$0.55(0.37-0.82)$

Risk categories

$$
\begin{aligned}
& \text { Low } \\
& \text { Medium } \\
& \text { High } \\
& >55 \\
& 55-64 \\
& 65-75 \\
& >75
\end{aligned}
$$

Referent

1.62 (1.09-2.40)

$2.51(1.67-3.76)$

Age

Referent

$1.60(0.61-4.18)$

$3.69(1.47-9.2)$

6.75 (2.65-17.92) the risk categories) were associated with survival (see Table 3). Type of treatment was associated with survival as well, with patients who underwent radical prostatectomy having higher survival compared to other types of treatment (results are not reported and available upon request). The difference in -2LL statistics was 476 for the model 
including HRQOL and 190 for the model without HRQOL Thus, $39.9 \%$ of the predictive information of the full multivariable model was contributed by HRQOL measures.

\section{Discussion}

In the study of the 2,899 men with localized prostate cancer, we examined both fixed (baseline and post-treatment) and time-varying association between HRQOL and survival. In the current study, patients reporting higher scores on several domains in SF-36 scales demonstrated lower likelihood of dying. In addition, those domains displayed a stable association with survival regardless of the period in treatment process, thus implying an important role of HRQOL in the survival in the patients with prostate cancer.

The associations found in our study are consistent with findings by other investigators; however, to our knowledge, it is the first study that investigated the relationship between HRQOL and survival in localized prostate cancer. In addition, results from our study demonstrated consistent association between HRQOL over time, underscoring importance of those outcomes in the course of disease and survivorship.

In the univariate analysis, higher scores on the majority of HRQOL domains were associated with better survival. Following the use of a sequential bootstrap technique, only physical function and general health demonstrated a strong association with survival. Two studies looking at association of HRQOL and survival in the patients with metastatic hormone-refractory prostate cancer showed similar results. In the study by Sullivan et al., hazard of death for patients whose baseline FACT-P grand total score was greater than median was $73 \%$ of the hazard for the patients whose baseline scores was less or equal to median [11]. In the study by Collette et al., only items of appetite loss and insomnia were retained as independent predictors [5]. Nevertheless, Collette and colleagues concluded that HRQOL measurements did not add to the predictive ability of the models. Similar results were reported in breast cancer populations $[8,13,28]$ although findings from the studies in non-metastatic cancer did not provide evidence of an association between baseline HRQOL and survival $[6,28]$.

Studies that evaluated the relationship between posttreatment assessments of HRQOL and survival reported mixed results. In the study by Coates et al., post-relapse scores were highly associated with survival, but were more pronounced in advanced disease [28]. In the study by Sullivan and colleagues, 12 week change scores were significant predictors of survival. Our results are consistent with these findings, demonstrating that change between baseline and the last assessment before the event in physical function, role physical, vitality, social function, and general health were highly associated with survival.

Addition of the time-dependent covariates to the conventional Cox proportional hazards models is thought to better reflect the dynamic nature of the disease. In the study by Lyyra et al., the addition of the time-dependent covariates led to the discovery of an association between selfreported health and survival while conventional analysis did not reveal those associations [16]. The study by KalantarZadeh and colleagues concluded that even though results from cross-sectional and time-dependent analysis of the association of osteodystrophy indicators in hemodialysis patients and survival were similar, subtle but potentially clinically relevant differences existed [29]. In our study, domains that were not initially identified in the conventional analysis were detected as an independently associated with survival. Relative risk for all-cause mortality was significantly lower for the patients reporting higher scores for role physical, physical function, and general health.

The mechanisms of the relationship between selfreported HRQOL and survival are not very well understood. Further investigation of these relationships could provide evidence of the pathways between physical and psychological manifestation of disease and might reflect patients' perceptions of the severity of underlying disease. A causal relationship between better HRQOL and survival has been suggested, although evidence is limited. One explanation of the predictive value of HRQOL is driven by the theory that patients are aware of the severity of the disease beyond the realm of conventional techniques (such as clinical characteristics), and this perception affects HRQOL self-reporting [28]. Moreover, it could be speculated that some domains of HRQOL might be a surrogate for possible symptoms and events, or HRQOL may be a marker for otherwise undetected prognostic factor.

Identification of prognostic factors for survival serves an important purpose. It could lead to modification of treatment regimens from aggressive to more palliative, as well as help identify subsets of patients for whom interventions could be applied. Because HRQOL is a multidimensional measure, it provides a more complete picture of patient's well-being beyond clinical characteristics. In addition, measures obtained during treatment might indicate a combined effect of the tumor response and an acute complication, and could be used as a supplemental parameter to modify the strategy for treatment. It should be noted that the importance of predictive value of HRQOL ought to be treated with caution. It has been shown that improvements in psychological support led to improvement in survival in breast cancer patients, as well as improvements in weight control [30]. However, until we can demonstrate that improvements in HRQOL could lead to improvement in survival after prostate cancer treatment, our findings are not necessarily causal. 
Several limitations exist in our study. Studies looking at the prognostic value of HRQOL used different instruments, making comparisons difficult. However, the majority of them demonstrated results in similar domains. In addition, it has been reported in several studies that comparability between different instruments is satisfactory. The risk of biases associated with use of an observational database that utilized non-random samples of the men are anticipated. Because our study concentrated on evaluation of the HRQOL over different time points, not all patients in the database have sufficient data, thus were not included in the analysis. Evaluation of the comorbidities was utilized by simple enumeration, which is a less than ideal measure of concurrent disease. However, we plan on utilizing expanded measures (TIBI-CaP) in future studies [31]. While race is considered to be an important factor that could influence survival, it was not evaluated based on the fact that more than $90 \%$ of the study population was white/ non-Hispanic, and inclusion of this variable would have reduce the power of the analysis.

While recognizing those limitations, our study has many advantages as well. Men in the CaPSURE ${ }^{\mathrm{TM}}$ database attend a geographically diverse group of mostly community-based practices and in general reflect treatment trends in the field. Longer follow-up and availability of baseline information on QOL allowed us to observe the effects of diagnosis, treatment, and survival over the natural course of disease. Several steps were taken to address effects of multicollinearity and the time-dependent nature of HRQOL. To our knowledge, it is the first study that evaluates association of HRQOL and survival in a large group of men with localized prostate cancer.

In conclusion, this study found that several domains of HRQOL were significantly associated with survival in a large group of patients with localized prostate cancer. These associations were maintained over the course of the disease regardless of the time of the assessment. Results from our study have both research and clinical relevance. They could provide us with information that would enable us to not only improve communication with patients and families, but to provide interventions and treatment best suitable for the patient.

Open Access This article is distributed under the terms of the Creative Commons Attribution Noncommercial License which permits any noncommercial use, distribution, and reproduction in any medium, provided the original author(s) and source are credited.

\section{References}

1. Brown, M. L., Lipscomb, J., \& Snyder, C. (2001). The burden of illness of cancer: Economic cost and quality of life. Annual Review of Public Health, 22, 91-113.
2. Health, Untied States. (2002). With chartbook on trends in the health of Americans. National Center for Health Statistics.

3. American Cancer Society. Cancer facts \& figs 2008. Atlanta: American Cancer Society.

4. Dharma-Wardene, M., Au, H. J., Hanson, J., et al. (2004). Baseline FACT-G score is a predictor of survival for advanced lung cancer. Quality of Life Research, 13, 1209-1216.

5. Collette, L., van Andel, G., Bottomley, A., et al. (2004). Is baseline quality of life useful for predicting survival with hormone-refractory prostate cancer? A pooled analysis of three studies of the European Organisation for Research and Treatment of Cancer Genitourinary Group. Journal of Clinical Oncology, 22, 3877-3885.

6. Efficace, F., Therasse, P., Piccart, M. J., et al. (2004). Healthrelated quality of life parameters as prognostic factors in a nonmetastatic breast cancer population: An international multicenter study. Journal of Clinical Oncology, 22, 3381-3388.

7. Fang, F. M., Tsai, W. L., Chiu, H. C., et al. (2004). Quality of life as a survival predictor for esophageal squamous cell carcinoma treated with radiotherapy. International Journal of Radiation Oncology, Biology, Physics, 58, 1394-1404.

8. Gupta, D., Granick, J., Grutsch, J. F., et al. (2007). The prognostic association of health-related quality of life scores with survival in breast cancer. Supportive Care in Cancer, 15, 387-393.

9. Herndon, J. E., I. I., Fleishman, S., Kornblith, A. B., et al. (1999). Is quality of life predictive of the survival of patients with advanced nonsmall cell lung carcinoma? Cancer, 85, 333-340.

10. Roychowdhury, D. F., Hayden, A., \& Liepa, A. M. (2003). Health-related quality-of-life parameters as independent prognostic factors in advanced or metastatic bladder cancer. Journal of Clinical Oncology, 21, 673-678.

11. Sullivan, P. W., Nelson, J. B., Mulani, P. M., et al. (2006). Quality of life as a potential predictor for morbidity and mortality in patients with metastatic hormone-refractory prostate cancer. Quality of Life Research, 15, 1297-1306.

12. Fang, F. M., Liu, Y. T., Tang, Y., et al. (2004). Quality of life as a survival predictor for patients with advanced head and neck carcinoma treated with radiotherapy. Cancer, 100, 425-432.

13. Efficace, F., Biganzoli, L., Piccart, M., et al. (2004). Baseline health-related quality-of-life data as prognostic factors in a phase III multicentre study of women with metastatic breast cancer. European Journal of Cancer, 40, 1021-1030.

14. Talcott, J. A., \& Clark, J. A. (2005). Quality of life in prostate cancer. European Journal of Cancer, 41, 922-931.

15. Litwin, M. S., Hays, R. D., Fink, A., et al. (1995). Quality-of-life outcomes in men treated for localized prostate cancer. JAMA, 273, 129-135.

16. Lyyra T. M., Leskinen E., Jylha M., et al. (2009). Self-rated health and mortality in older men and women: A time-dependent covariate analysis. Archives of Gerontology and Geriatrics, 48, $14-18$.

17. Ferraro, K. F., \& Kelley-Moore, J. A. (2001). Self-rated health and mortality among black and white adults: Examining the dynamic evaluation thesis. The Journals of Gerontology. Series B, Psychological Sciences and Social Sciences, 56, S195-S205.

18. Han, B., Phillips, C., Ferrucci, L., et al. (2005). Change in selfrated health and mortality among community-dwelling disabled older women. Gerontologist, 45, 216-221.

19. Van Steen, K., Curran, D., Kramer, J., et al. (2002). Multicollinearity in prognostic factor analyses using the EORTC QLQC30: Identification and impact on model selection. Statistics in Medicine, 21, 3865-3884.

20. Sauerbrei, W., \& Schumacher, M. (1992). A bootstrap resampling procedure for model building: Application to the Cox regression model. Statistics in Medicine, 11, 2093-2109. 
21. Lubeck, D. P., Litwin, M. S., Henning, J. M., et al. (1996). The CaPSURE database: A methodology for clinical practice and research in prostate cancer. CaPSURE Research Panel. Cancer of the Prostate Strategic Urologic Research Endeavor. Urology, 48, 773-777.

22. Cooperberg, M. R., Broering, J. M., Latini, D. M., et al. (2004). Patterns of practice in the United States: Insights from CaPSURE on prostate cancer management. Current Urology Reports, 5, $166-172$.

23. Ware, J. E., Jr., \& Sherbourne, C. D. (1992). The MOS 36-item short-form health survey (SF-36). I. Conceptual framework and item selection. Medical Care, 30, 473-483.

24. D'Amico, A. V., Whittington, R., Malkowicz, S. B., et al. (2002). Biochemical outcome after radical prostatectomy or external beam radiation therapy for patients with clinically localized prostate carcinoma in the prostate specific antigen era. Cancer, 95, 281-286.

25. Messinger-Rapport, B., Pothier Snader, C. E., Blackstone, E. H., et al. (2003). Value of exercise capacity and heart rate recovery in older people. Journal of the American Geriatrics Society, 51, 63-68.
26. Fisher, L. D., \& Lin, D. Y. (1999). Time-dependent covariates in the Cox proportional-hazards regression model. Annual Review of Public Health, 20, 145-157.

27. Allison, P. D. (1995). Survival analysis using the SAS system: A practical guide. Cary, NC: SAS Institute Inc.

28. Coates, A. S., Hurny, C., Peterson, H. F., et al. (2000). Quality-oflife scores predict outcome in metastatic but not early breast cancer. International Breast Cancer Study Group. Journal of Clinical Oncology, 18, 3768-3774.

29. Kalantar-Zadeh, K., Kuwae, N., Regidor, D. L., et al. (2006). Survival predictability of time-varying indicators of bone disease in maintenance hemodialysis patients. Kidney International, 70, $771-780$

30. Courneya, K. S., Mackey, J. R., Bell, G. J., et al. (2003). Randomized controlled trial of exercise training in postmenopausal breast cancer survivors: Cardiopulmonary and quality of life outcomes. Journal of Clinical Oncology, 21, 1660-1668.

31. Litwin, M. S., Greenfield, S., Elkin, E. P., et al. (2007). Assessment of prognosis with the total illness burden index for prostate cancer: Aiding clinicians in treatment choice. Cancer, 109, $1777-1783$. 